\title{
Evaluation of Lower-Dose Spiral Head CT for Detection of Intracranial Findings Causing Neurologic Deficits
}

\author{
(D).G. Fletcher, (DD.R. DeLone, (D) A.L. Kotsenas, (D) N.G. Campeau, (D).T. Lehman, (DL. Yu, (D). Leng, (DD.R. Holmes III,
} (D)P.K. Edwards, (DM.P. Johnson, (D) G.J. Michalak, DR.E. Carter, and (D) C.H. McCollough

\begin{abstract}
BACKGROUND AND PURPOSE: Despite the frequent use of unenhanced head CT for the detection of acute neurologic deficit, the radiation dose for this exam varies widely. Our aim was to evaluate the performance of lower-dose head CT for detection of intracranial findings resulting in acute neurologic deficit.
\end{abstract}

\begin{abstract}
MATERIALS AND METHODS: Projection data from 83 patients undergoing unenhanced spiral head CT for suspected neurologic deficits were collected. Cases positive for infarction, intra-axial hemorrhage, mass, or extra-axial hemorrhage required confirmation by histopathology, surgery, progression of findings, or corresponding neurologic deficit; cases negative for these target diagnoses required negative assessments by two neuroradiologists and a clinical neurologist. A routine dose head CT was obtained using 250 effective $\mathrm{mAs}$ and iterative reconstruction. Lower-dose configurations were reconstructed (25-effective mAs iterative reconstruction, 50-effective mAs filtered back-projection and iterative reconstruction, 100-effective mAs filtered back-projection and iterative reconstruction, 200-effective mAs filtered back-projection). Three neuroradiologists circled findings, indicating diagnosis, confidence (0-100), and image quality. The difference between the jackknife alternative free-response receiver operating characteristic figure of merit at routine and lower-dose configurations was estimated. A lower $95 \% \mathrm{Cl}$ estimate of the difference greater than -0.10 indicated noninferiority.
\end{abstract}

RESULTS: Forty-two of 83 patients had 70 intracranial findings (29 infarcts, 25 masses, 10 extra- and 6 intra-axial hemorrhages) at routine head CT (CT dose index $=38.3 \mathrm{mGy}$ ). The routine-dose jackknife alternative free-response receiver operating characteristic figure of merit was $0.87(95 \% \mathrm{Cl}, 0.81-0.93)$. Noninferiority was shown for 100-effective $\mathrm{mAs}$ iterative reconstruction (figure of merit difference, $-0.04 ; 95 \% \mathrm{Cl},-0.08$ to 0.004$)$ and 200 -effective mAs filtered back-projection $(-0.02 ; 95 \% \mathrm{Cl},-0.06$ to 0.02$)$ but not for 100 -effective mAs filtered back-projection $(-0.06 ; 95 \% \mathrm{Cl},-0.10$ to -0.02$)$ or lower-dose levels. Image quality was better at higher-dose levels and with iterative reconstruction $(P<.05)$.

CONCLUSIONS: Observer performance for dose levels using 100-200 eff mAs was noninferior to that observed at 250 effective $\mathrm{mAs}$ with iterative reconstruction, with iterative reconstruction preserving noninferiority at a mean CT dose index of $15.2 \mathrm{mGy}$.

ABBREVIATIONS: $\mathrm{CTDI}_{\text {vol }}=$ volume $\mathrm{CT}$ dose index; eff. $\mathrm{mAs}=$ effective $\mathrm{mAs} ; \quad \mathrm{FBP}=$ filtered back-projection; $\mathrm{GEE}=$ generalized estimating equation; $\mathbb{R}=$ iterative reconstruction; JAFROC FOM = jackknife alternative free-response receiver operating characteristic figure of merit

U nenhanced head CT is frequently requested in the emergency department or inpatient setting to examine patients with suspected neurologic deficit as well as those having

Received March 29, 2019; accepted after revision August 21.

From the Departments of Radiology (J.G.F., D.R.D., A.L.K., N.G.C., V.T.L., L.Y., S.L., G.J.M., C.H.M.), Biomedical Imaging Resource (D.R.H., P.E.), and Biomedical Statistics and Informatics (M.P.J.), Mayo Clinic, Rochester, Minnesota; and Health Sciences Research (R.E.C.), Mayo Clinic, Jacksonville, Florida.

The project described was supported by the National Institutes of Health grant No. U01 EB17185.

The content is solely the responsibility of the authors and does not necessarily represent the official views of the National Institutes of Health.

Paper previously presented, in part, at: Scientific Assembly and Annual Meeting of the Radiological Society of North America, November 27 to December 2, 2016; Chicago, Illinois. undergone recent trauma. Surprisingly, the technique is not standardized, and the radiation dose varies substantially among institutions. ${ }^{1}$ Radiologists strive to acquire CT examinations at the lowest dose that will answer the diagnostic question in accordance with the as low as reasonably achievable principle ${ }^{2}$ and the justification that the diagnostic benefit to the patient

Please address correspondence to Joel G. Fletcher, MD, Mayo Clinic, Department of Radiology, 200 First St SW, Rochester, MN 55905; e-mail: fletcher.joel@mayo.edu

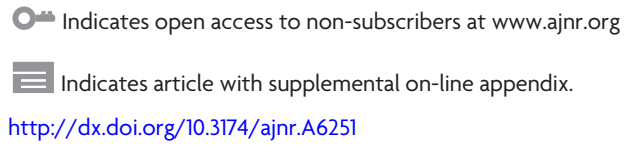

AJNR Am J Neuroradiol 40:1855-63 Nov 2019 www.ajnr.org 
outweighs the small theoretic risk $^{3,4}$ of associated radiation injury. Unfortunately, trade-offs between observer performance and radiation dose are not well-delineated, even for most common CT tasks, and this dearth of information likely contributes to the differences in doses among institutions.

When one uses CT to answer very specific clinical questions, dramatic dose reduction for high-contrast detection tasks using unenhanced head CT can be undertaken (eg, to rule out craniosynostosis or shunt check). ${ }^{5-7}$ However, there are scarce scientific data or an established consensus for the lowest acceptable dose for head CT for the general evaluation of suspected neurologic deficit. CT detection of early acute cerebral infarction, subtle intracranial hemorrhage, or intracranial masses is a more challenging and demanding diagnostic task because these pathologies result in only subtle low-contrast differences in the involved structures. Iterative reconstruction (IR) can provide noninferior performance for high-contrast detection tasks at lower doses; however, recent data in phantoms and abdominal CT suggest that the improvement in observer performance compared with filtered back-projection (FBP) may be limited..$^{8-11}$

Most studies examining lower-dose head CT with iterative reconstruction have relied on subjective comparisons of image quality or contrast-to-noise ratios rather than observer (ie, radiologist) performance. Practical considerations such as collecting cases with proven imaging findings, obtaining CT images at multiple doses, and correlating imaging findings between dose levels and reference standards make such research challenging.

We have recently studied a small number of patients $(n=43)$ with suspected neurologic deficit. ${ }^{12,13}$ This preliminary study defined lower-dose levels that were unable to preserve observer performance but included a mix of both subtle and very obvious cases, which may have affected our ability to discriminate observer performance among varying dose levels. The current study builds on these initial results to compare observer performance between routine and lower-dose levels for the detection of visually challenging intracranial findings causing acute neurologic deficit in a larger number of patients and addresses the ability of iterative reconstruction to assist with dose reduction.

\section{MATERIALS AND METHODS \\ Patients and Cases}

The primary diagnostic task for this study was to identify imaging findings that may require further treatment or evaluation or that may potentially explain patient signs or symptoms in patients with suspected acute neurologic deficit. After approval by the institutional review board of Mayo Clinic, we archived CT image and projection data of patients who underwent clinically indicated spiral head CT examinations for suspected acute neurologic deficit and who provided consent for the use of medical records for research purposes. CT image and projection data were collected between August 20, 2013, and May 3, 2014. Archived CT images were then reviewed by a board-certified reference neuroradiologist (D.R.D., with 19 years of experience as an attending neuroradiologist) to ensure that inclusion and exclusion criteria were met for this retrospective, case-control study.
All patients underwent CT for suspected acute neurologic deficit. Inclusion criteria were different for positive and negative cases but required reference criteria to be met. Positive cases were additionally required to have sufficient clinical evidence to meet reference standard criteria for at least 1 of 4 target conditions causing acute neurologic deficit: 1) infarction (acute, subacute, chronic, or indeterminate age), 2) intra-axial hemorrhage (eg, contusion), 3) mass, or 4) extra-axial hemorrhage (eg, subdural, subarachnoid, epidural, intraventricular). Reference standard criteria for accepting positive cases into the study required confirmation of an imaging abnormality on the CT examination by the reference radiologist, in addition to the following: 1) clinical physician confirmation of neurologic deficits at physical examination corresponding to abnormal imaging findings on the index CT examination, 2) progression or confirmation of imaging findings on another imaging examination (eg, subsequent MR imaging, CT perfusion), or 3) confirmation of imaging findings at an operation. The reference neuroradiologist, unblinded to all clinical information and all subsequent imaging and surgical reports, then marked CT images for all CT findings relating to the target diagnosis that were present within the imaged volume that met inclusion criteria using a specially designed computer workstation, tightly circumscribing each CT imaging finding relating to each diagnosis and documenting correlative reference information using standard menus. An ROI was also placed within white or gray matter (as appropriate) to reflect the background CT number in which the target lesion was located.

Negative cases were required to have sufficient evidence that a suspected acute neurologic deficit was not present. Reference standard criteria for accepting negative cases into the study included both the clinical neuroradiologist interpreting the head $\mathrm{CT}$ at the time of imaging as well as the unblinded reference neuroradiologist indicating that no imaging findings associated with the 4 target conditions were present, and lack of focal neurologic findings on physical examination by the clinical attending neurologist. Imaging findings of small vessel ischemic change associated with aging (leukoaraiosis) were noted in all included CT exams whether or not one of the four target diagnoses were present.

To determine the lowest radiation dose at which observer performance was noninferior to routine dose, we constructed the study cohort to display visually challenging imaging findings that might affect a radiologist's ability to detect the target diagnoses at different radiation dose levels. Obvious CT findings such as a large intracranial hemorrhage can be detected at even the $10 \%-$ $20 \%$ dose levels, ${ }^{12}$ and inclusion of such obvious cases does not help discriminate the diagnostic differences among dose levels. The nonreader reference neuroradiologist visually evaluated 857 CT examinations (620 negative and 237 positive) that met all reference and inclusion criteria and graded the conspicuity of abnormal imaging findings associated with the target diagnoses along a 4-point scale: 1) minimally evident (eg, minimal obscuration of the lentiform nucleus and insular cortex in new infarcts that might be easily missed), 2) subtle (eg, more definite acute infarct or small metastases on noncontrast spiral head CT), 3) distinct abnormality with well-defined borders (eg, small chronic infarct, small intraparenchymal hematoma), or 4) obvious 
857 patients undergoing spiral head CT for suspected

neurologic deficit with archived CT projection data

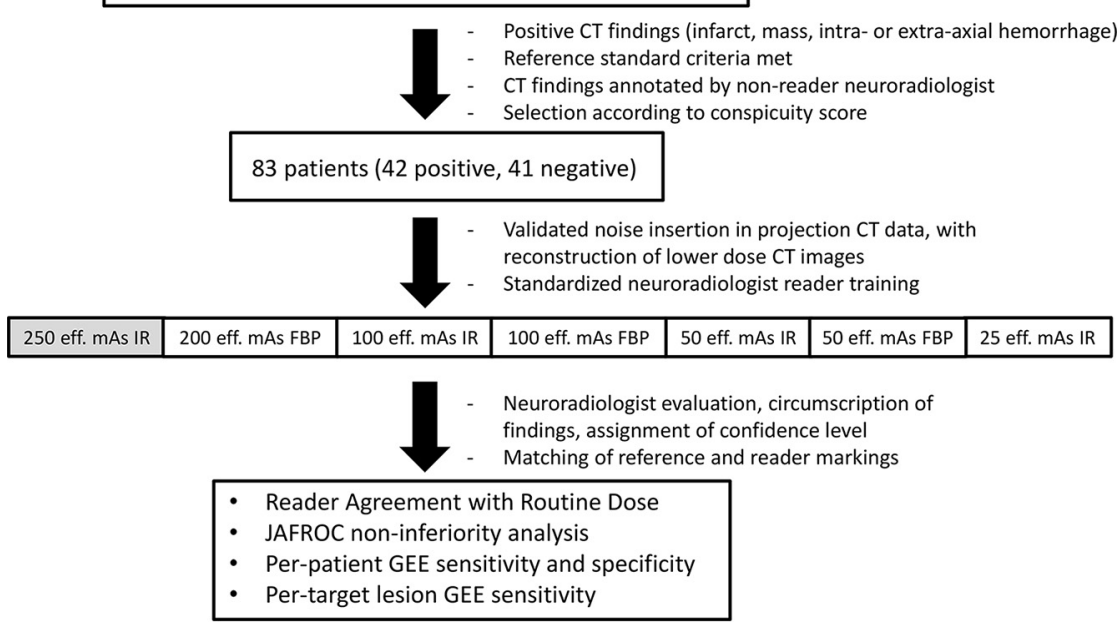

FIG 1. Study schema.

Table 1: Dose levels and reconstruction kernels for unenhanced CT examinations used in this study

\begin{tabular}{lcc}
\hline $\begin{array}{c}\text { Tube } \\
\text { Current } \\
\text { Setting }\end{array}$ & $\begin{array}{c}\text { CTDI }_{\text {vol }} \\
\text { (mGy) }\end{array}$ & $\begin{array}{c}\text { Reconstruction } \\
\text { Kernel } \\
\text { (Type, Strength) }\end{array}$ \\
\hline 250 eff. mAs & 38.1 & J40 (IR 2) \\
200 eff. mAs & 30.5 & H40 (FBP) \\
100 eff. mAs & 15.2 & J40 (IR 2) \\
100 eff. mAs & 15.2 & H40 (FBP) \\
50 eff. mAs & 7.6 & J40 (IR 2) \\
50 eff. mAs & 7.6 & H40 (FBP) \\
25 eff. mAs & 3.8 & J40 (IR 2) \\
\hline
\end{tabular}

finding (eg, chronic territorial infarct, large acute intraparenchymal hematoma, or diffuse subarachnoid hemorrhage). Thus, cases with obvious imaging findings that would have no discriminatory value in selecting the appropriate radiation dose level would not be included. On the basis of this subjective conspicuity score, visually challenging positive cases that met reference criteria were selected from among the collected positive cases meeting inclusion criteria (Fig 1).

The target population for this study was constructed as previously described, with a population of 83 patients, approximately half of whom would have 1 of the 4 target lesions causing acute neurologic deficit. ${ }^{12}$

\section{Image Acquisition and Reconstruction}

Unenhanced spiral head CT examinations were acquired using nearly identical CT platforms (Definition FLASH or Definition AS+; Siemens, Erlangen, Germany) using a single x-ray tube, a detector configuration of $64 \times 0.6 \mathrm{~mm}$ with a $\mathrm{z}$-flying focal spot yielding 128 detector rows, 1 -second tube rotation time, $120 \mathrm{kV}$ (peak), and either 250 effective mAs (eff. mAs) or 340 eff. mAs. The higher tube current was obtained if the examination was part of a trauma scan in our emergency department. Routinedose CT images were reconstructed using a J40 head kernel using iterative reconstruction with a strength of 2 (sinogram-affirmed iterative reconstruction [SAFIRE]; Siemens), with 5-mm-thick images reconstructed every $5 \mathrm{~mm}$ in the axial plane and 2-mm-thick images reconstructed every $2 \mathrm{~mm}$ in the coronal plane. CT images corresponding to lower-dose levels were created by inserting image noise in CT projection data using a highly accurate and validated noise-insertion tool. ${ }^{5,6,13,14}$ For projection data obtained using $120 \mathrm{kV}$ and 340 eff. mAs, noise was inserted to obtain images corresponding to 250 eff. mAs so that all patient "routine" dose examinations corresponded to the same dose level. On the basis of the prior results, CT projection data dose levels corresponding to 100,50 , and 25 eff. mAs were then also created using iterative reconstruction at these dose levels, and additionally with FBP at 50, 100, and 200 eff. mAs (Table 1). Noise-inserted CT projection data for each case were subsequently loaded back onto the CT system to reconstruct corresponding axial and coronal images with the appropriate reconstruction kernel.

\section{Image Evaluation by Neuroradiologists}

Three neuroradiologists with 18,18 , and 5 years of experience as clinical neuroradiologists at our institution were selected as blinded radiologist readers. Because of the unique features of head CT (complicated anatomy, variety of normal aging processes not representing pathology), a standardized reader training manual that defined pathologies to be detected and instructions for reporting reader confidence scores (with anchors) was developed and reviewed by each participating neuroradiologist (On-line Appendix). Confidence scores ranged from 0 (indicating certainty that the circumscribed finding is not one of the target lesions) to 100 (indicating the highest degree of certainty that can be achieved with CT for one of the target findings). ${ }^{15,16}$ Neuroradiologists were instructed not to mark frequently seen aging processes such as small-vessel ischemic change (leukoaraiosis), benign intraparenchymal calcification, chronic lacunar infarctions, or arachnoid cysts. Formal one-on-one reader training with the principal investigator was completed, with each reader interpreting 20 training cases selected to match the case mix, pathologies, and dose-reconstruction configurations in the subsequent reader study, discussing reader confidence ratings and any questions. ${ }^{17}$

Readers evaluated routine-dose-reconstruction and 6 lowerdose-reconstruction configurations using a specialized computer workstation viewing images using at least 2 window settings (80/40 and 33/40) in multiple sessions. Once a CT finding corresponding to a target diagnosis was identified, readers were instructed to tightly circumscribe all imaging abnormalities 


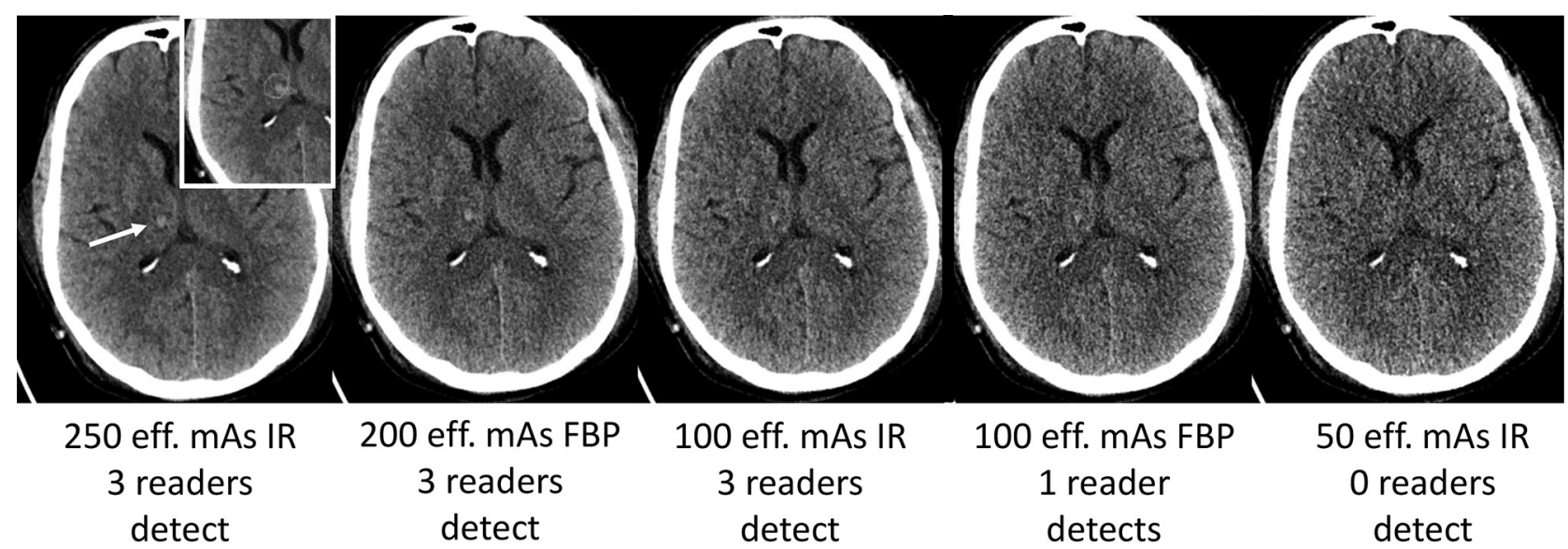

FIG 2. Small right thalamic hemorrhage (white arrow) shown on routine-dose CT image (250-eff. mAs IR) along with lower-dose configurations. The small left inset shows reference neuroradiologist markings of the target lesion (green circle). This CT examination was performed after trauma, with hemorrhage confirmed surgically, and the final diagnosis was recorded as right thalamic hemorrhage consistent with shear injury.

corresponding to one of the target diagnoses using a spline tool. Readers rated their level of confidence that one of the target diagnoses was present. Subsequently, readers answered 4 image-quality questions related to overall quality, image sharpness, image noise, and noise texture based on the modified European Quality Criteria, with overall image quality rated along a 5-point scale (1, nondiagnostic due to excessive noise or artifacts; 2 , diagnosis questionable due to excessive noise or artifacts, moderate decrease in confidence; 3 , diagnostic with moderate-but-acceptable noise or artifacts; 4 , mild noise, no change in confidence; and 5, routine diagnostic image quality). ${ }^{14,18}$ Examinations were interpreted in random order, with only 1 dose-reconstruction configuration per patient interpreted during each session.

\section{Statistical Analysis}

The sample size for this study was determined as a part of a 3stage study design with the objective in this stage to screen and prioritize imaging strategies for evaluation in a large, future 10reader, multicase study (stage 3). Stage 1, which consisted of 43 independent cases, has been previously published. ${ }^{12}$ The original sample size calculations determined that 83 cases were needed for this second stage of the study.

Matching of colocalized reference and reader detections was performed by the reference neuroradiologist (Fig 2). Comparison of reader performance between routine-dose head CT (at 250 eff. mAs) using a head IR kernel (J40 strength 2) with lower-dose reconstruction configurations was performed using reader agreement rules and jackknife alternative free-response receiver operating characteristic figure of merit (JAFROC FOM) noninferiority analysis. Reader agreement rules compared reader localizations on the routine dose with the lower-dose configurations. For positive cases (i.e., one of the four target diagnoses was present), readers had to localize all target lesions, which were identified by 2 of 3 neuroradiologist readers at routine-dose configurations (denoted as "essential lesions"). For negative cases without imaging findings corresponding to a focal neurologic deficit, no false-positive localizations could be made by $\geq 2$ of the neuroradiologist readers. Preset criteria for prioritization of a lower-dose configuration were agreement with routine dose interpretation in 71 of the 83 cases ( $86 \%$ of examinations). ${ }^{8}$

JAFROC FOM analysis used the reader confidence scores input by the readers as well as their circumscribed imaging findings. A full description of JAFROC FOM for a mixed population as in this study has been provided previously. ${ }^{19,20}$ When $>1$ imaging finding was present in positive cases, findings were weighted according to the reciprocal of the number of findings. FOMs were calculated for every dose level and every reader. The contrasts (comparisons) of FOMs were estimated using the Hillis improvement (Dorfman, Berbaum, and Metz method under the modeling assumption of fixed readers, random cases using the Rjafroc package, Version 1.0.1 [https://cran.r-project.org/]). Noninferiority of lower-dose configurations was represented by calculating the estimated difference between routine and lower-dose configurations, with the limit of noninferiority set at -0.10 , a value determined a priori on the basis of investigator consensus. This limit means that when the lower limit of the $95 \%$ confidence interval is greater than -0.10 , noninferiority with observer performance at a routine dose is achieved.

In addition to these analyses, typical measures of diagnostic accuracy such as per-patient sensitivity and specificity and per-lesion sensitivity were performed. For the purposes of this calculation, a cutoff reader confidence of 10 (of 100) for one of the target findings was used. For per-patient specificity, there could be no reader circumscriptions with a confidence level of $>10$ in negative cases. For these measures of diagnostic performance, generalized estimating equations (GEEs) were used to estimate the pooled estimate across the 3 readers for each imaging strategy.

For the image quality ratings (eg, overall impression of diagnostic image quality), a summary score was computed for each of the datasets. The summary score was the mean of the 3 readers. Tests for differences in image quality across dose and reconstruction were facilitated by a mixed model consisting of a random patient factor to account for the correlation of image qualities across the doses and assumed Gaussian errors. Post hoc 
Table 2: Reference documentation and conspicuity of proved lesions in positive CT examinations with imaging findings corresponding to cause of acute neurologic deficit $(n=42)$

\begin{tabular}{|c|c|c|c|}
\hline $\begin{array}{c}\text { Target } \\
\text { Diagnosis }\end{array}$ & $\begin{array}{l}\text { No. of Imaging } \\
\text { Findings with } \\
\text { Target Diagnosis }\end{array}$ & No. with Reference Criterion (Nonexclusive List) & $\begin{array}{l}\text { Ranking of Conspicuity } \\
\text { Scores }^{\mathrm{a}} \\
\text { (Mean) (SD) }\end{array}$ \\
\hline Infarct & 29 & $\begin{array}{l}\text { Clinical confirmation of corresponding deficit }=29 \\
\text { Progression/confirmation on another imaging study }=23 \\
\text { Confirmation at surgery }=0\end{array}$ & $2.10(0.76)$ \\
\hline Mass & 25 & $\begin{array}{l}\text { Clinical confirmation of corresponding deficit }=22 \\
\text { Progression/confirmation on another imaging study }=21 \\
\text { Confirmation at surgery }=2\end{array}$ & $2.80(0.70)$ \\
\hline $\begin{array}{l}\text { Extra-axial } \\
\text { hemorrhage }\end{array}$ & 10 & $\begin{array}{l}\text { Clinical confirmation of corresponding deficit }=10 \\
\text { Progression/confirmation on another imaging study }=3 \\
\text { Confirmation at surgery }=0\end{array}$ & $2.60(0.66)$ \\
\hline $\begin{array}{l}\text { Intra-axial } \\
\text { hemorrhage }\end{array}$ & 6 & $\begin{array}{l}\text { Clinical confirmation of corresponding deficit }=6 \\
\text { Progression/confirmation on another imaging study }=2 \\
\text { Confirmation at surgery }=0\end{array}$ & $2.67(0.47)$ \\
\hline
\end{tabular}

a Please see Materials and Methods. In brief, conspicuity scores: 1, minimally evident; 2, subtle; 3, distinct focal abnormality; 4, obvious.

Table 3: Reader agreement of lower-dose reconstruction configurations compared with routine-dose unenhanced head CT examinations, along with JAFROC FOMs ${ }^{\mathrm{a}}$

\begin{tabular}{|c|c|c|c|c|c|c|}
\hline \multirow[b]{2}{*}{$\begin{array}{l}\text { Lower-Dose- } \\
\text { Reconstruction } \\
\text { Configuration }\end{array}$} & \multicolumn{2}{|c|}{$\begin{array}{c}\% \text { of the } 47 \text { Essential } \\
\text { Lesions }{ }^{b} \text { Detected } \\
\text { by Readers at } \\
\text { Lower-Dose } \\
\text { Configurations } \\
\end{array}$} & \multicolumn{3}{|c|}{$\begin{array}{l}\text { No. of Successful Interpretations per Lower- } \\
\text { Dose-Reconstruction Configuration }\end{array}$} & \multirow[b]{2}{*}{$\begin{array}{l}\text { JAFROC FOM } \\
(95 \% \mathrm{CI})\end{array}$} \\
\hline & 2 of 3 & 3 of 3 & $\begin{array}{l}\text { Cases with at } \\
\text { Least } 1 \\
\text { Essential Lesion } \\
\quad(n=34)\end{array}$ & $\begin{array}{l}\text { Cases without } \\
\text { Any Essential } \\
\text { Lesions } \\
(n=49)\end{array}$ & $\begin{array}{c}\text { No. Successful } \\
\text { Interpretations } \\
\text { ( } \geq 71 \text { Required } \\
\text { per Design) }\end{array}$ & \\
\hline $\begin{array}{l}200 \text { eff. mAs } \\
\text { FBP }\end{array}$ & $44(94 \%)$ & $39(83 \%)$ & 30 & 48 & 78 & $0.846(0.78-0.912)$ \\
\hline 100 eff. mAs IR & $43(92 \%)$ & 37 (79\%) & 29 & 46 & 75 & $0.831(0.764-0.898)$ \\
\hline 100 eff. mAs FBP & $42(89 \%)$ & $36(77 \%)$ & 28 & 45 & 73 & $0.805(0.732-0.878)$ \\
\hline 50 eff. mAs IR & 41 (87\%) & $32(68 \%)$ & 26 & 47 & 73 & $0.795(0.727-0.864)$ \\
\hline 50 eff. mAs FBP & $38(81 \%)$ & 31 (66\%) & 25 & 47 & 72 & $0.789(0.717-0.861)$ \\
\hline 25 eff. mAs IR & $34(72 \%)$ & $25(53 \%)$ & 22 & 45 & $67^{c}$ & $0.754(0.681-0.827)$ \\
\hline
\end{tabular}

comparisons of the quality summary score were considered descriptive and were not adjusted for multiple testing across doses. We evaluated the effect of certain CT lesion characteristics such as size, CT number difference, and contrast-to-noise ratio compared with adjacent normal-appearing brain parenchyma on observer performance using Spearman rank coefficients comparing these parameters with mean reader confidence for correctly detected lesions. For false-negative examinations, a confidence score of zero was assigned.

\section{RESULTS}

Eighty-three CT examinations in patients with suspected neurologic deficit had a mean volume CT dose index $\left(\mathrm{CTDI}_{\mathrm{vol}}\right)$ of $38.1 \pm 1.3 \mathrm{mGy}$. Forty-two positive cases had 70 proven target lesions (ie, mean, $1.6 \pm 1.2$ target imaging findings causing suspected neurologic deficit per patient). There were 29 infarcts, 25 masses, 10 extra-axial hemorrhages, and 6 intra-axial hemorrhages (Table 2). The mean area for the imaging findings was
$6.9 \pm 8.2 \mathrm{~cm}^{2}$, with the longest and shortest linear dimensions being $3.9 \pm 3.0 \mathrm{~cm}$ and $2.2 \pm 1.1 \mathrm{~cm}$, respectively. Thirty patients (36\%) had leukoaraiosis (17 with proven target lesions, 13 without lesions).

Table 3 shows reader agreement for lower-dose configurations along with JAFROC FOMs. The 25-eff. mAs IR configuration failed to meet preset criteria for reader agreement rules. For lower-dose configurations, a greater proportion of nonagreement with the routine dose comes from missed lesions (false-negative interpretations) rather than false-positive interpretations (or localizations) in negative examinations (Figs 2 and 3). At least 2 of 3 of the neuroradiologists identified $94 \%$ of the target lesions at 200 -eff. mAs FBP, and this declined to $87 \%$ at 50 -eff. mAs IR and $81 \%$ at 50 -eff. $\mathrm{mAs}$ FBP, while the number of correctly interpreted negative cases remained virtually identical.

Table 4 shows the GEE per-patient sensitivity and specificity, as well as the target lesion sensitivity for CT findings accounting for neurologic deficits in our patient 


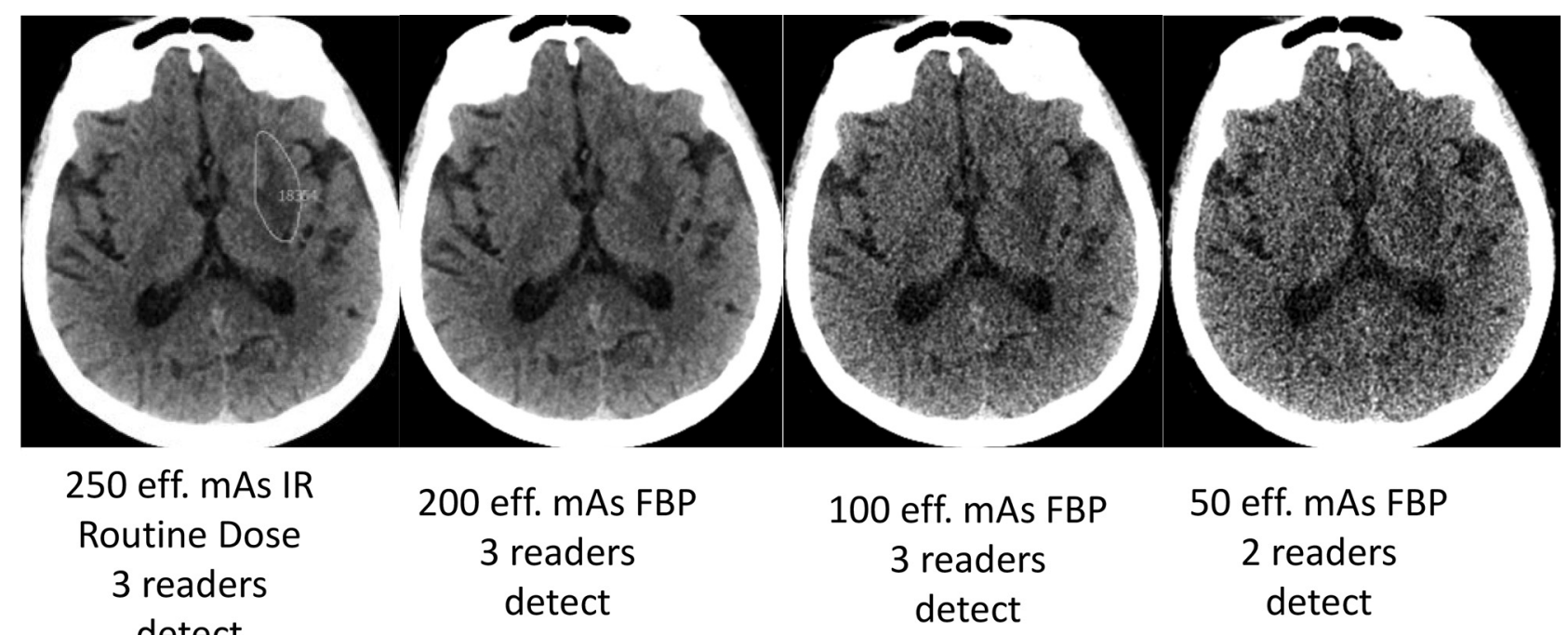

FIG 3. Acute left lentiform nucleus infarct (green circle indicates reference neuroradiologist markings at routine dose) with corresponding lower-dose FBP CT images along with reader results. The imaging finding on this CT examination evolved with time, with corresponding clinical confirmation of corresponding neurologic deficit by a staff neurologist, and the final diagnosis was recorded as acute left striatal infarct.

Table 4: Per-patient and per-lesion sensitivity and specificity using GEEs for target neurologic findings accounting for acute neurologic deficits

\begin{tabular}{lccc}
\multicolumn{1}{c}{$\begin{array}{c}\text { Dose-Kernel } \\
\text { Configuration }\end{array}$} & $\begin{array}{c}\text { Per-Patient Sensitivity for CT } \\
\text { Findings Accounting for Acute } \\
\text { Neurologic Deficits (GEE) (\%) } \\
(95 \% \text { CI) (Range) (\%) }\end{array}$ & $\begin{array}{c}\text { Per-Patient Specificity for CT } \\
\text { Findings Accounting for Acute } \\
\text { Neurologic Deficits (GEE) (\%) } \\
(95 \% \text { CI) (Range) (\%) }\end{array}$ & $\begin{array}{c}\text { Target Lesion Sensitivity for CT } \\
\text { Findings Accounting for Acute } \\
\text { Neurologic Deficits (GEE) (\%) } \\
(95 \% \text { CI) (Range) (\%) }\end{array}$ \\
\hline 250-eff. mAs IR & $81.7(71.1-92.3)(78.6-83.3)$ & $93.5(88.9-98.1)(85.4-100.0)$ & \\
200-eff. mAs FBP & $79.4(68.2-90.6)(76.2-83.3)$ & $91.9(87.5-96.3)(80.5-100.0)$ & $68.6(62.3-74.9)(61.4-72.9)$ \\
100-eff. mAs IR & $77.0(65.5-88.5)(73.8-81.0)$ & $88.6(82.8-94.4)(73.2-95.1)$ & $68.1(61.8-74.4)(64.3-71.4)$ \\
100-eff. mAs FBP & $74.6(62.4-86.8)(69.0-78.6)$ & $87.0(81.1-92.9)(75.6-95.1)$ & $62.9(56.3-69.4)(57.1-65.7)$ \\
50-eff. mAs IR & $73.8(62.3-85.4)(66.7-78.6)$ & $88.6(82.8-94.4)(75.6-97.6)$ & $60.5(53.9-67.1)(52.9-65.7)$ \\
50-eff. mAs FBP & $72.2(60.6-83.8)(71.4-73.8)$ & $83.7(77.7-89.8)(69.8-86.0)$ & $61.0(54.4-67.6)(60.0-62.9)$ \\
25-eff. mAs IR & $65.9(53.3-78.4)(61.9-71.4)$ & $88.6(82.4-94.8)(85.4-92.7)$ & $53.3\left(46.6-60.1(45.7-62.9)^{\mathrm{a}}\right.$ \\
\hline
\end{tabular}

${ }^{a}$ The $95 \%$ confidence interval does not overlap the routine dose, so the dose-reconstruction configuration is significantly worse.

population. Per-target lesion GEE sensitivity was significantly decreased compared with routine-dose unenhanced head CT for a 25-eff. mAs IR configuration (Figs 4 and 5). Per-patient GEE sensitivity was not significantly different from the reference dose at any lower configurations. GEEs per patient specificity declined only slightly at lower doses.

Figure 4 is the forest plot demonstrating the difference between routine-dose and the lower-dose configurations. Only the 100-eff. mAs IR and 200-eff. mAs FBP lower-dose configurations demonstrated noninferiority compared with the routine dose, with noninferiority not demonstrated for 100-eff. mAs FBP and lower-dose configurations.

Figure 5 demonstrates the overall image-quality ratings for routine and lower-dose configurations. Each lower-dose configuration had significantly inferior image quality $(P<.05)$. At 100 -eff. mAs and 50-eff. mAs dose levels, configurations with iterative reconstruction had significantly higher image quality $(P<.001)$.

Mean reader confidence was moderately affected by lesion size (Spearman correlation coefficients for lower-dose configurations: $p=0.23, P=.053$ for FBP 200 eff. mAs and $p=0.35-$
$0.39, P<.05$ for lower doses). Mean reader confidence had a somewhat weaker relationship with both the contrast-to-noise ratio ( $p=0.16, P=.18$ for FBP 200 eff. $m A$ s and $p=0.24-0.32$, $P<.05$ for lower doses) and the CT number difference ( $p=0.11, P=.35$ at FBP 200 eff. $\mathrm{mAs}$ and $p=0.2-0.29$ for lower doses with $P<.05$ at FBP 100 eff. mAs, FBP 50 eff. mAs, and IR 25 eff. mAs).

\section{DISCUSSION}

In keeping with the as low as reasonably achievable principle, radiologists strive to perform diagnostically useful imaging at the lowest possible radiation exposure dose to the patient. In this work, we systematically evaluated the ability to reduce the radiation dose for spiral unenhanced head CT for acute neurologic deficit without compromising neuroradiologist observer performance. Our study cohort included patients with suspected neurologic deficit with either proven CT findings positive for infarction, intra-axial hemorrhage, mass, or extra-axial hemorrhage by the reference criteria or CT examinations negative for imaging findings correlating to these target diagnoses accompanied by lack of focal neurologic findings on physical 


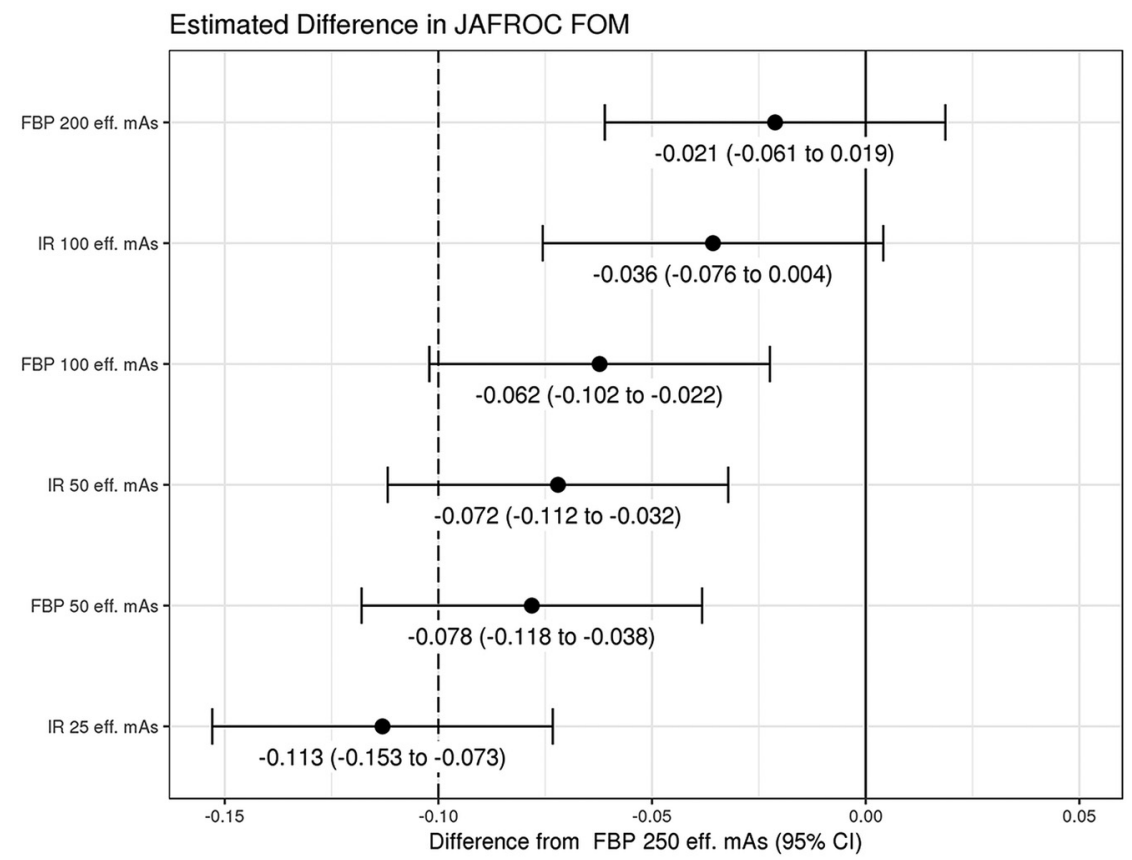

FIG 4. Noninferiority analysis showing the difference between JAFROC FOM at a routine dose and the lower-dose configurations for CT findings causing acute neurologic deficit. The limit of noninferiority was established a priori to be -0.10 , meaning that if the lower limit of the $95 \%$ confidence interval is greater than -0.10 , then noninferiority was shown.
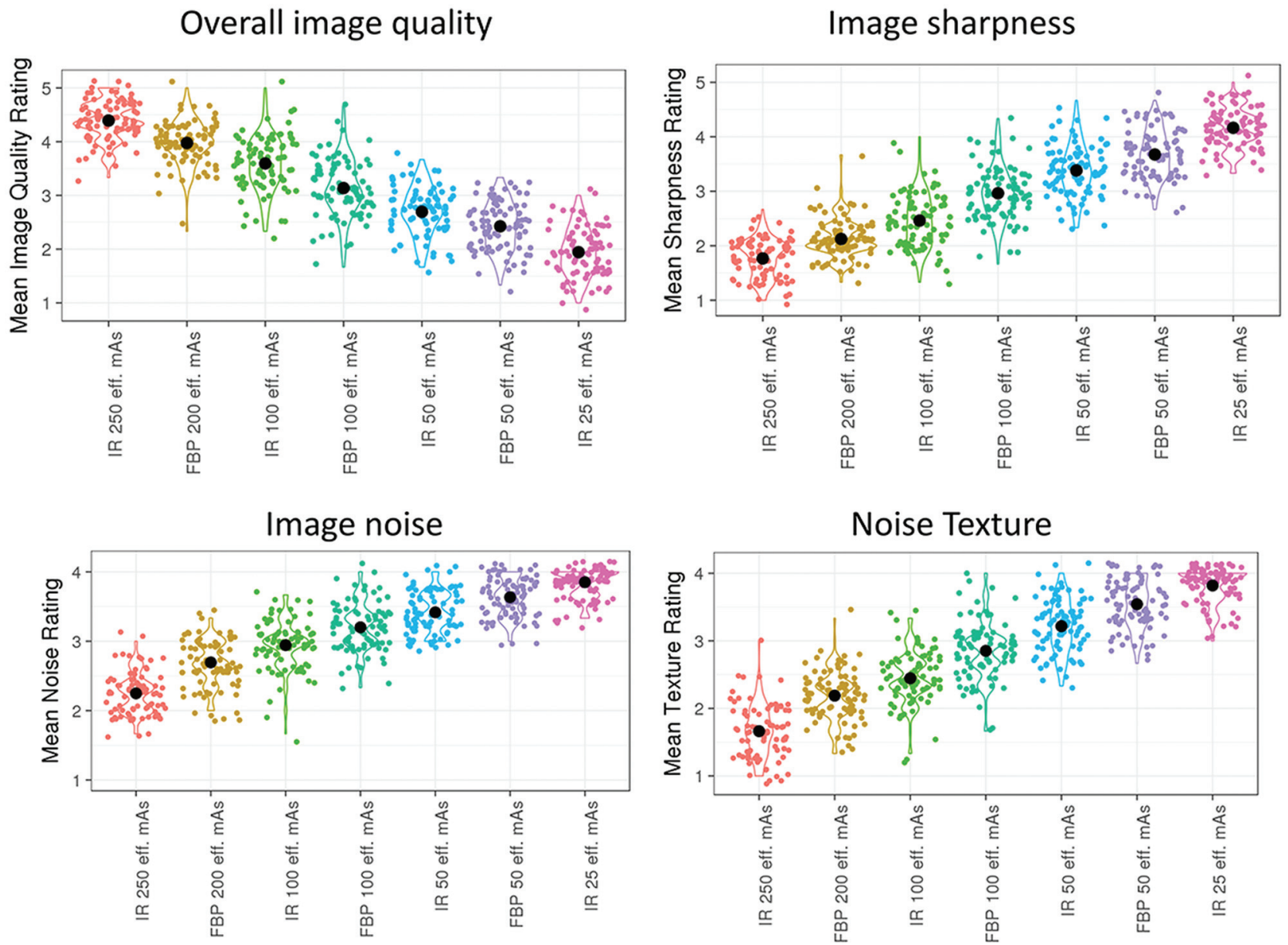

FIG 5. Image-quality metrics for routine and lower-dose configurations in this study. Optimal ratings were 5 for image quality and 1 for individual image metrics (i.e. image sharpness, noise, and texture). 
examination by the clinical attending neurologist. We found that there were small, nonsignificant decreases in sensitivity for CT findings accounting for neurologic deficits at all lower-dose configurations but that there was substantial opportunity for dose reduction if small decreases in performance were acceptable. Specifically, we found that observer performance was noninferior down to $40 \%$ of our routine dose level (ie, a tube current setting of 100 effective mAs, corresponding to a $\mathrm{CTDI}_{\mathrm{vol}}$ of $15.2 \mathrm{mGy}$; Table 4) when iterative reconstruction was used. At the same dose level with images reconstructed with weighted FBP, sensitivity for target lesions accounting for neurologic deficits declined by about $2 \%-3 \%$ and specificity declined by $1.5 \%-5 \%$.

Prior work examining the ability to lower the radiation dose at unenhanced head CT has been largely focused on the ability to improve image quality, ${ }^{21-23}$ because this may be a barrier to radiologists examining noisier, lower-dose images. $\mathrm{Wu}$ et $\mathrm{al}^{24}$ examined image quality and diagnostic accuracy using iterative reconstruction with a case-control design and lowering the rotation dose by $43 \%$ with either tube current or tube voltage reduction. They found that dose reduction achieved with tube current reduction and IR preserved image quality, but their study did not report observer performance for pathologic entities, relying instead on detection of subcortical arteriosclerotic encephalopathy and the number of infarcts as a surrogate marker. Additionally, several studies evaluating unenhanced head CT have not used spiral head CT, which can facilitate a lower radiation dose compared with sequential scanning. ${ }^{25}$ Without observer performance data to ensure that lower doses do not result in inferior performance, routine diagnostic levels are often set by subjective image-quality perceptions of individuals or by benchmarking to other institutions. Having observer performance data on which to facilitate dose reduction is needed for making objective decisions and may assist in overcoming differences among institutions.

Recent clinical and phantom studies in abdominal CT have highlighted the limitations of iterative reconstruction and its ability to facilitate radiation-dose reduction for low-contrast objects such as hepatic metastases. Mileto et $\mathrm{al}^{9}$ recently had 16 radiologists examine a low-contrast detectability phantom and found that radiation doses resulted in similar declines in observer performance for FBP and IR and that differences among radiologists were larger than across reconstruction algorithms. Jensen et $\mathrm{al}^{11}$ found that an approximately $50 \%$ dose reduction with one iterative reconstruction approach did not preserve observer performance for the detection of hepatic metastases. Fletcher et $\mathrm{al}^{8,12}$ found that FBP and IR performed similarly at lower doses in detecting hepatic metastases and that IR might only be needed to maintain observer performance at a certain threshold dose. Similar to these studies, we found that performance for the detection of intracranial findings causing acute neurologic deficits declined slightly at lower doses using JAFROC FOM as a metric of observer performance but that iterative reconstruction was helpful in preserving noninferiority at 100 eff. mAs. On the basis of this work, we plan to refine the results with a multireader, multcase study with 10 neuroradiologists to better predict the lowest dose that can preserve performance.
Our study has limitations. Because of the concern for missing subtle findings in patients with acute neurologic deficits, we used a retrospective case-control study design using an enriched cohort of visually challenging CT findings to discriminate radiation dose levels, with these findings proven on the basis of surgical assessment, follow-up imaging, or corresponding neurologic deficits. From a radiation exposure standpoint, it is not possible to re-image patients directly at multiple differing exposures during the same imaging session. Therefore, we relied on a validated noise-insertion method to reconstruct CT images corresponding to multiple dose levels; however, we have found that this method is highly accurate and has allowed our clinical practice to readily adopt research findings obtained using this method. ${ }^{5,6} \mathrm{CT}$ interpretation at reduced radiation dose levels is subjectively more demanding and fatiguing than at standard doses, and the potential effects of radiologist fatigue could not be measured in our study because neuoradiologists interpreted cases with different dose levels in each interpretation session. Finally, our results relied on interpretations by only 3 neuroradiologists, and extrapolation of their interpretations to a larger number of neuroradiologists or general radiologists may be limited.

\section{CONCLUSIONS}

This study helps to better define the potential dose reduction that can be achieved with conventional spiral CT and IR that will maintain diagnostic performance for evaluation of suspected neurologic deficit. Our study demonstrates that substantial opportunity exists for dose reduction using spiral nonenhanced head CT and that the dose level might potentially be reduced to $40 \%$ of routine dose levels or a CTDI $\mathrm{vol}_{\mathrm{vo}}$ of approximately $15 \mathrm{mGy}$ if slight decreases in performance are acceptable (eg, in follow-up and surveillance). Furthermore, the beneficial effect of IR was most pronounced at this $15-\mathrm{mGy}$ dose level. Above this dose level, observer performance might be preserved with filtered back-projection alone.

\section{ACKNOWLEDGMENTS}

We acknowledge Maria Shiung, Adam Bartley, and Kris Nunez for their dedicated assistance in carrying out this study and preparing the manuscript. We appreciate the support of Dr Kent Thielen, Chair of the Department of Radiology at Mayo Clinic Rochester, for his commitment to ensure that a large number of staff could participate in this endeavor over a long period of time.

Disclosures: Joel G. Fletcher-UNRELATED: Grants/Grants Pending: Siemens.* David R. Holmes-RELATED: Grant: National Institutes of Health.* Cynthia H. McCollough-UNRELATED: Grants/Grants Pending: Siemens, Comments: Industry Research Grant.* *Money paid to the institution.

\section{REFERENCES}

1. Smith-Bindman R, Wang Y, Yellen-Nelson TR, et al. Predictors of $\mathrm{CT}$ radiation dose and their effect on patient care: a comprehensive analysis using automated data. Radiology 2017;282:182-93 CrossRef Medline 
2. Centers for Disease Control and Prevention. ALARA: As Low As Reasonably Achievable. https://www.cdc.gov/nceh/radiation/alara. html. Accessed February 22, 2019

3. McCollough CH, Bushberg JT, Fletcher JG, et al. Answers to common questions about the use and safety of CT scans. Mayo Clin Proc 2015;90:1380-92 CrossRef Medline

4. McCollough CH, Guimaraes L, Fletcher JG. In defense of body CT. AJR Am J Roentgenol 2009;193:28-39 CrossRef Medline

5. Gabriel S, Eckel LJ, DeLone DR, et al. Pilot study of radiation dose reduction for pediatric head $\mathrm{CT}$ in evaluation of ventricular size. AJNR Am J Neuroradiol 2014;35:2237-42 CrossRef Medline

6. Montoya JC, Eckel LJ, DeLone DR, et al. Low-dose CT for craniosynostosis: preserving diagnostic benefit with substantial radiation dose reduction. AJNR Am J Neuroradiol 2017;38:672-77 CrossRef Medline

7. Widmann G, Schullian P, Gassner EM, et al. Ultralow-dose CT of the craniofacial bone for navigated surgery using adaptive statistical iterative reconstruction and model-based iterative reconstruction: 2D and 3D image quality. AJR Am J Roentgenol 2015;204:56369 CrossRef Medline

8. Fletcher JG, Fidler JL, Venkatesh SK, et al. Observer performance with varying radiation dose and reconstruction methods for detection of hepatic metastases. Radiology 2018;289:455-64 CrossRef Medline

9. Mileto A, Zamora DA, Alessio AM, et al. CT detectability of small low-contrast hypoattenuating focal lesions: iterative reconstructions versus filtered back projection. Radiology 2018;289:443-54 CrossRef Medline

10. Goenka AH, Herts BR, Obuchowski NA, et al. Effect of reduced radiation exposure and iterative reconstruction on detection of low-contrast low-attenuation lesions in an anthropomorphic liver phantom: an 18-reader study. Radiology 2014;272:154-63 CrossRef Medline

11. Jensen CT, Wagner-Bartak NA, Vu LN, et al. Detection of colorectal hepatic metastases is superior at standard radiation dose CT versus reduced dose CT. Radiology 2019;290:400-09 CrossRef Medline

12. Fletcher JG, Yu L, Fidler JL, et al. Estimation of observer performance for reduced radiation dose levels in CT: eliminating reduced dose levels that are too low is the first step. Acad Radiology 2017; 24:876-90 CrossRef Medline

13. Yu L, Shiung MM, Jondal D, et al. Development and validation of a practical lower-dose-simulation tool for optimizing CT scan protocols. J Comput Assist Tomogr 2012;36:477-87 CrossRef Medline

14. Fletcher JG, Yu L, Li Z, et al. Observer performance in the detection and classification of malignant hepatic nodules and masses with
CT image-space denoising and iterative reconstruction. Radiology 2015;276:465-78 CrossRef Medline

15. Briggs $M$, Closs JS. A descriptive study of the use of visual analogue scales and verbal rating scales for the assessment of postoperative pain in orthopedic patients. J Pain Symptom Manage 1999;18:43846 CrossRef Medline

16. Hadjiiski L, Chan HP, Sahiner B, et al. Quasi-continuous and discrete confidence rating scales for observer performance studies: effects on ROC analysis. Acad Radiol 2007;14:38-48 CrossRef Medline

17. Dendumrongsup T, Plumb AA, Halligan S, et al. Multi-reader multicase studies using the area under the receiver operator characteristic curve as a measure of diagnostic accuracy: systematic review with a focus on quality of data reporting. PLoS One 2014;9:e116018 CrossRef Medline

18. Froemming AT, Kawashima A, Takahashi N, et al. Individualized $\mathrm{kV}$ selection and tube current reduction in excretory phase computed tomography urography: potential for radiation dose reduction and the contribution of iterative reconstruction to image quality. J Comput Assist Tomogr 2013;37:551-59 CrossRef

19. Chakraborty D, Yoon H. JAFROC analysis revisited: figure-ofmerit considerations for human observer studies. Proceedings of SPIE 2009;7263:72630T CrossRef Medline

20. Chakraborty D, Philips P, Zhai X, et al. RJafroc: Analyzing Diagnostic Observer Performance Studies. https:/CRAN.Rproject.org/package=RJafroc. Accessed January 14, 2019

21. Harris M, Huckle J, Anthony D, et al. The acceptability of iterative reconstruction algorithms in head CT: an assessment of sinogram affirmed iterative reconstruction (SAFIRE) vs. filtered back projection (FBP) using phantoms. J Med IMag Rad Sci 2017;48:259-69 CrossRef Medline

22. Korn A, Fenchel M, Bender B, et al. Iterative reconstruction in head CT: image quality of routine and low-dose protocols in comparison with standard filtered back-projection. AJNR Am J Neuroradiol 2012;33:218-24 CrossRef Medline

23. Love A, Olsson ML, Siemund $\mathrm{R}$, et al. Six iterative reconstruction algorithms in brain CT: a phantom study on image quality at different radiation dose levels. Br J Radiol 2013;86:20130388 CrossRef Medline

24. Wu TH, Hung SC, Sun JY, et al. How far can the radiation dose be lowered in head CT with iterative reconstruction? Analysis of imaging quality and diagnostic accuracy. Eur Radiol 2013;23:261221 CrossRef Medline

25. Pace I, Zarb F. A comparison of sequential and spiral scanning techniques in brain CT. Radiol Technol 2015;86:373-78 Medline 